\title{
Subglottic stenosis in paediatric patients
}

\author{
Santosh Kumar Swain', Mahesh Chandra Sahu², Jasashree Choudhury ${ }^{3}$ \\ 'Department of Otorhinolaryngology, IMS and SUM Hospital, Siksha "O" Anusandhan University, K8, Kalinganagar, \\ Bhubaneswar, Odisha, India \\ 2Directorate of Medical Research, IMS and SUM Hospital, Siksha "O" Anusandhan University, K8, Kalinganagar, Bhubaneswar, \\ Odisha, India \\ ${ }^{3}$ Department of Paediatrics, IMS and SUM Hospital, Siksha "O" Anusandhan University, K8, Kalinganagar, Bhubaneswar, \\ Odisha, India
}

\section{ABSTRACT}

Subglottic stenosis (SGS) is a complex and challenging clinical entity for paediatric otolaryngologists. Subglottic stenosis is a congenital or acquired lesion characterised by narrowing of the airway extending from the lower free border of the vocal folds to the lower border of the cricoid cartilage. The most common cause of SGS is prolonged endotracheal intubation. The treatment option for SGS is often surgery, which may be endoscopic or open. Nowadays, the endoscopic approach is reaching its limit, and open surgery is advised only in failed cases of endoscopic approach. In this review article, we discuss the current concept and recent advances in the diagnosis and treatment of SGS.

\section{KEY WORDS:}

subglottic stenosis, paediatric patient, endoscopic approach, laryngotracheal surgery.

\section{INTRODUCTION}

Subglottic stenosis (SGS) is defined as narrowing of the subglottis, which is a part of the larynx extending from the lower margin of the true vocal cords to the inferior border of the cricoid cartilage [1]. Subglottic stenosis can be an acquired clinical entity occuring after prolonged intubation or a congenital condition leading to respiratory distress during the neonatal period. There has been significant advancement in the investigations and treatment of the SGS in children. The management of the SGS needs a team approach, which includes a paediatric otolaryngologist, anaesthesiologist, paediatrician, pulmonologist, and intensive care specialist. The incidence of SGS is around 1 to $2 \%$ among intubated neonates and $11 \%$ of the intubated children below five years old [2]. The rate of SGS increases by $50 \%$ for every five days of intubation in children below five years old [3]. A premature baby can tolerate prolonged intubation better than a term baby. This review article focuses on the etiopathogenesis, clinical presentations, and current management of SGS in children, with special emphasis given to the clinical experiences.

\section{ETIOPATHOGENESIS OF SUBGLOTTIC STENOSIS}

The aetiopathogenesis of SGS is complex. The aetiology of congenital SGS is attributed to the developmental failure during pregnancy and is often associated with congenital head and neck lesions and syndromes like 22q11deletion, Down syndrome, and CHARGE syndrome [4]. Acquired SGS is often due to prolonged ventilation at the intensive care unit. The subglottis of the larynx is vulnerable to intubation trauma due to its

\section{ADDRESS FOR CORRESPONDENCE:}

Santosh Kumar Swain, Department of Otorhinolaryngology, IMS and SUM hospital, Siksha "O" Anusandhan

University, K8, Kalinganagar, Bhubaneswar 751003, Odisha, India, e-mail: santoshvoltaire@yahoo.co.in 
anatomical features. It is the narrowest part of the larynx and is the only part that is surrounded by a complete cartilaginous ring, not allowing insertion of the endotracheal tube. The most common and dangerous complication of children with prolonged endolaryngeal intubation is SGS. The incidence of SGS in intubated infant is in the range of 1 to $10 \%$ [5]. Ulcerations are usually seen after endolaryngeal intubation. The depth of the ulcerations varies from superficial to full-thickness. Regeneration of the epithelium is usually seen at the subglottis and is seen as squamous metaplasia. This leads to fibrous scar tissue formation leading in turn to stenosis. There are a number of causes for vulnerability to injury and stenosis of the subglottis in children. First, the respiratory mucosal lining of the subglottis is delicate and is easily injured by the endotracheal tube. Second, the cricoid is a complete cartilage ring, so oedema occurs by direct trauma by the tip of the endotracheal tube or pressure impingement from the inflated cuff on the internal diameter of the subglottis region [6]. Third, the loose areolar tissue at the submucosa of the subglottis can develop significant oedema rapidly after endotracheal injury. Fourth, the subglottis is the narrowest region of the airway in children. Attention should be paid to the endotracheal tube size, cuff pressure, and avoidance of tube movement by nasotracheal intubation, resulting in less injury to the subglottis. Other causes of SGS are high tracheostomy causing injury to the cricoid cartilage, emergency cricothyroidotomy, caustic ingestion, excessive laser, smoke inhalation, trauma, and burn. SGS can also be caused by tumours like subglottic haemangioma and less commonly by fibroma or chondroma. Chronic inflammatory conditions like Wegner's granulomatosis, relapsing polychondritis, amyloidosis, pemphigoid, major aphthous ulcer, and laryngocutaneous syndrome can cause SGS. Chronic diseases like tuberculosis or syphilis can also lead to SGS.

\section{CLINICAL PRESENTATIONS}

The clinical manifestations of SGS are directly related to the degree of stenosis. Grade I SGS is often asymptomatic, but the symptoms are aggravated during upper airway infections with respiratory distress and even stridor. Grade III and IV SGS often present with air hunger, dyspnoea, biphasic stridor, suprasternal, intercostals, and diaphragmatic retractions. Recurrent episodes of croup may give rise to suspicion of SGS. It is often important for the clinician or paediatrician to urgently identify a compromised airway that might lead to rapid deterioration and needs prompt intervention to avoid a catastrophic situation. Extubation failure in a child often excludes the non-laryngeal causes like nasal obstruction, glossoptosis, and tracheobronchomalacia for respiratory distress. It is always advisory to document difficult intubation, history of prolonged intubation, as well as failed extubation, which give clues as to the cause of SGS. If there is any past history of airway surgery, it should always be documented with the type and timing of surgery. The clinical conditions that cause stridor are the differential diagnosis of SGS. These clinical situations are croup, subglottic haemangioma, subglottic cyst, vocal cord paralysis, complete tracheal rings, and bacterial tracheitis. Congenital SGS is the third most common congenital lesion of the larynx [7]. Subglottic stenosis is considered as congenital when there is no history of endotracheal intubation or any acquired causes of SGS. The clinical presentations of congenital SGS are directly related to the degree of narrowing of the subglottis. In severe congenital SGS, stridor is present at the time of birth, whereas in less severe, the symptoms are seen after few months of birth. Congenital SGS is less common than acquired and can only be diagnosed before its first intubation in child.

\section{GRADING OF SUBGLOTTIC STENOSIS}

The classical grading of SGS (Fig. 1) was described by Cotton and approved worldwide. The modified Cotton Myer grading (Fig. 2) based on the percentage of obstruction calculated on the basis of passing an endotracheal tube through the stenosis, resulting in the approximate diameter of stenotic segment divided by the age-appropriate endotracheal size [8]. There are some disadvantages of this grading system. Vocal cord status has an impact on the treatment of SGS. The vocal cord immobility with grade I stenosis will not provide an adequate airway. Other factors like length of SGS have an impact on the treatment. A severe SGS with a thin scar web is usually treated easily by endoscopic method, whereas a long but a less narrow segment is often resistant to endoscopic ap-

\begin{tabular}{|c|c|}
\hline Classification & From \\
\hline Grade I & \\
\hline Grade II & No obstruction \\
\hline Grade III & N1\% obstruction \\
\hline Grade IV & \\
\hline
\end{tabular}

FIGURE 1. Cotton Meyer grading of SGS 


\begin{tabular}{|c|c|c|c|c|c|c|c|c|c|c|}
\hline Patient & & & & Percentag & e of Obstruc & tion Actual E & ndotracheal & Tube Size: & & \\
\hline Age & & $I D=2.0$ & $I D=2.5$ & $I D=3.0$ & $I D=3.5$ & $I D=4.0$ & $\mathrm{ID}=4.5$ & $I D=5.0$ & $I D=5.5$ & $I D=6.0$ \\
\hline & & no obstruction & & & & & & & & \\
\hline Premature & & 40 & no obstruction & & & & & & & \\
\hline & No & 58 & 30 & no obstruction & & & & & & \\
\hline $0-3 \mathrm{mo}$ & Detectable & 68 & 48 & 26 & no obstruction & & & & & \\
\hline 3-9 mo & Lumen & 75 & 59 & 41 & 22 & no obstruction & & & & \\
\hline $9 \mathrm{mo}-2 \mathrm{yr}$ & & 80 & 67 & 53 & 38 & 20 & no obstruction & & & \\
\hline $2 \mathrm{yr}$ & & 84 & 74 & 62 & 50 & 35 & 10 & no obstruction & & \\
\hline $4 \mathrm{yr}$ & & 86 & 78 & 68 & 57 & 45 & 32 & 17 & no obstruction & \\
\hline $6 \mathrm{yr}$ & & 89 & 81 & 73 & 64 & 54 & 43 & 30 & 16 & no obstruction \\
\hline & Grade IV & & Grade III & & Gra & de II & & & de I & \\
\hline
\end{tabular}

FIGURE 2. Modified Cotton Meyer grading of SGS

proach. The consistency of SGS, e.g. granulation tissue or scar tissue, also has an impact on the treatment.

\section{DIAGNOSIS}

The diagnosis of SGS (Fig. 3) is done by proper history taking and physical examination of the child. History should be taken regarding birth injury, prolonged intubation, prematurity, and other congenital anomalies, and physiologic indicators of respiratory or cardiovascular status should be documented. The duration and onset of stridor should be documented. The type of stridor, e.g. inspiratory, expiratory, and biphasic, should also be documented. The voice quality of the child is also important to give an idea of the location of the pathology [9]. Feeding problems should be documented. History of extubation failure or non-laryngeal causes like nasal obstruction, glossoptosis, and tracheobronchomalacia should also be docu-

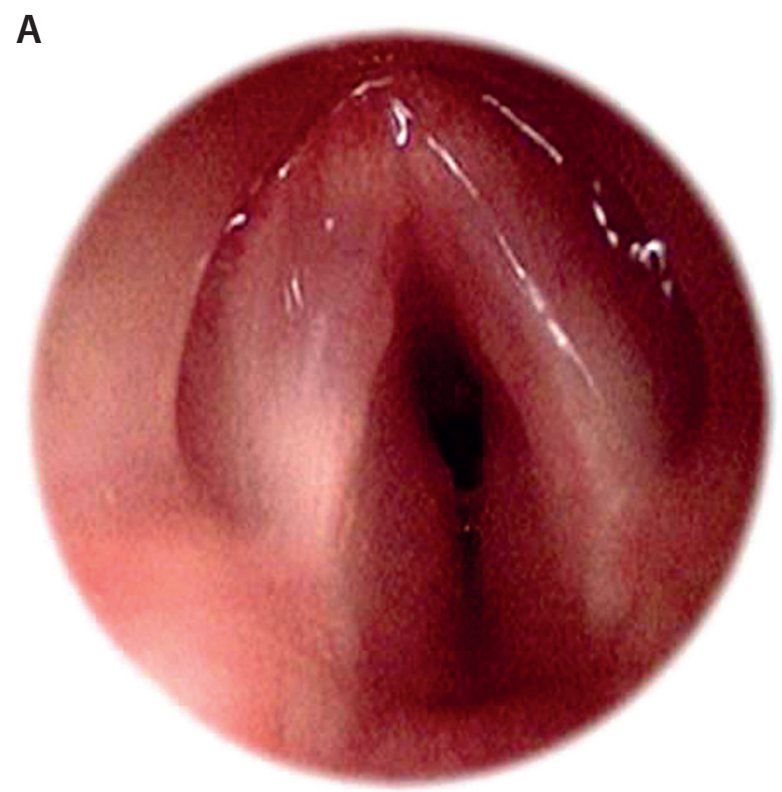

FIGURE 3. Endoscopic picture showing SGS mented. History of difficult intubation or failed extubations supports laryngotracheal stenosis. The situation, like the current ventilator requirement of the child, determines whether early reconstruction is needed or not. Any history of endoscopic or open airway surgery should be documented, including timing and reasons for failure of the surgery. In the case of tracheostomised children (Fig. 4), the size of the tracheostomy tube, tolerance to the speech valve, and the possibility of tracheostomy tube plugging should be documented. The clinical conditions causing stridor should be considered for differential diagnosis, like croup, subglottic haemangioma, subglottic cyst, bacterial tracheitis, vocal cord paralysis, and complete tracheal rings.

\section{IMAGING}

The imaging evaluation provides detailed information regarding the site and length of the stenotic segment at

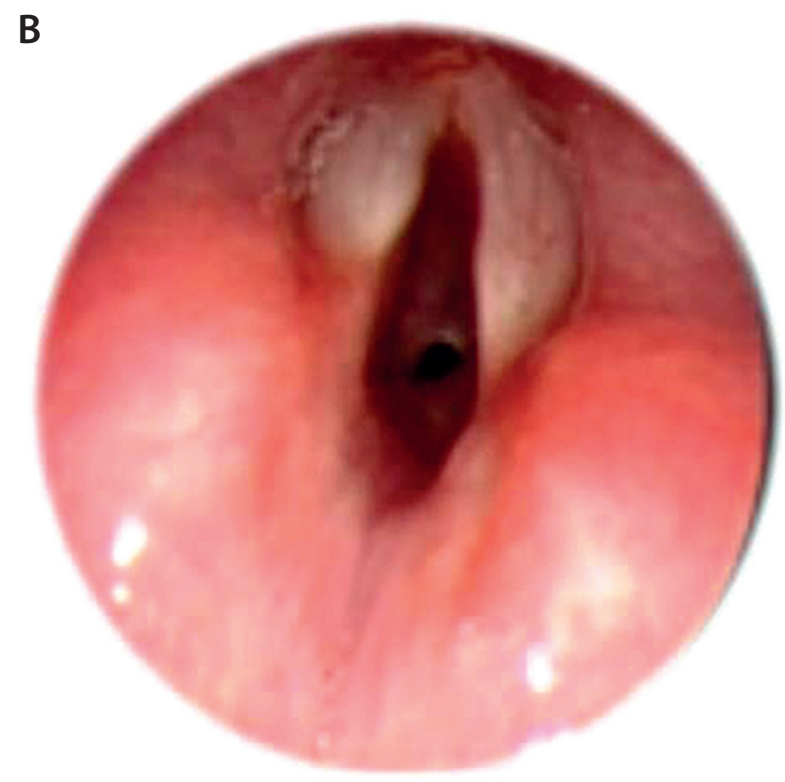


the airway. The diagnosis of the SGS is usually confirmed by direct laryngoscopy. A routine computed tomography (CT) scan or magnetic resonance imaging (MRI) is advised for most children with SGS. Magnetic resonance angiography (MRA) is usually done when suspected for vascular anomaly or tumour causing airway obstruction. An MRI or CT scan is done when direct laryngoscope reveals near complete or complete obstruction at the subglottis, to assess the length of the stenotic segment [10].

\section{ENDOSCOPIC EVALUATION}

The gold standard evaluation for laryngotracheal airway stenosis is endoscopic assessment. Rigid bronchoscopy and microlaryngoscopy will identify the site and degree of stenosis from larynx to carina. If a patient has a tracheostomy tube, attention should be paid to the suprastomal area to rule out any suprastomal collapse, suprastomal granuloma, and any skin tract. The distal and middle tracheal assessment rules out any complete tracheal rings, a tracheoesophageal fistula, vascular compression, ortracheomalacia. The carina and main bronchi should be examined.

\section{TREATMENT}

The treatment on SGS is based on history, severity of the clinical presentations (breathing difficulty), age of the child, vocal cord mobility, previous intervention, co-morbidities like developmental, neurological, and aspiration, and other associated airway lesions. Taking the above considerations along with direct laryngoscopy findings and an imaging report, the appropriate decision is taken for treatment of SGS. A child presenting with respiratory distress in the emergency room require surgent attention and intervention. A sign of impending disaster is increased effort of breathing despite normal oxygen saturation. In this situation, the child may suddenly decompensate. Here, a quick decision is needed for secure airway. The safest area in which to provide a secured airway is the operating room, where direct laryngoscopy or rigid bronchoscopy can be done in the case of difficult intubation. A tracheostomy set should be ready for emergent tracheostomy if the above procedures are unsuccessful. Because the airway of the child is collapsible, cricothyroidotomy is unsafe and is not a suitable procedure, so it should be avoided in children. Inhalation nebulisation with adrenaline and use of heliox may help to reduce breathing difficulties before intervention to provide a secure airway. Children with SGS with grade I or mild grade II without tracheostomy may not need surgery and can be observed [11]. In children with very mild stridor, never hospitalised for imminent airway compromise. As children have reactive larynx, watchful waiting and avoiding surgery those have tracheostomy. A larynx is referred to as "reactive" when it is associated with inflammation and granulations. Paediatric

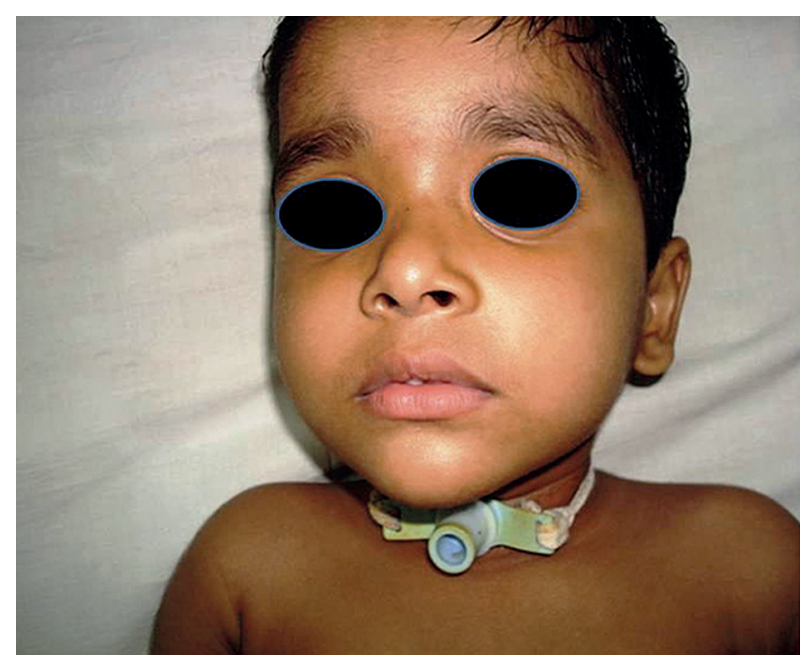

FIGURE 4. Child with SGS who has undergone tracheostomy

patients tend to heal poorly after surgery, so open airway reconstructive surgery should be avoided until the larynx is no longer "reactive". Laryngotracheal reconstructive surgery is contraindicated in: children with low weight, e.g. less than $10 \mathrm{~kg}$, recurrent aspiration pneumonia, children with neurological lesions with high potential for recurrent aspiration, and those with severe pulmonary disease [11].

\section{MEDICAL TREATMENT}

Medical treatment is useful in a few selected cases. Intravenous or inhaled corticosteroids are indicated if SGS is diagnosed early. It influences the inflammatory process at the site of the lesion. Beclomethasone aerosol every six hours improves the patient's symptoms. Intravenous hydrocortisone prevents suprastomal granulations [12].

\section{SURGICAL TREATMENT}

The two surgical options in SGS are: endoscopic or open. Endoscopic surgery is usually helpful in the treatment of grade I or II. Grade III or IV SGS need open surgical treatment like laryngotracheal reconstructions (LTR), laryngotracheoplasty (LTP), or cricotracheal resection (CTR). But nowadays, with the advancement of technology, the endoscopic approach has reached its limit, and open surgery is only done in case of failed cases of endoscopic approach.

\section{MICRODEBRIDER}

The microdebrider is usually used in endoscopic sinus surgery but may also be suitable for laryngeal endoscopic sinus surgery by using skimmer blades [13]. The microdebrider is used in cases of laryngeal papillomatosis, and it can be used in subglottic granuloma or fibroma, haemangioma, and subglottic cysts. The advantage of the microdebrider is its accuracy of tissue excision without damaging adjacent tissue. 


\section{BALLOON DILATION}

Balloon dilation is an effective endoscopic surgical option and it is an alternative to open reconstructive surgery. It has a $66 \%$ success rate in preventing tracheostomy or LTR [14]. It has also a relatively low complication rate. Serial balloon dilation is a safe and effective endoscopic method for treating SGS.

\section{BOUGIE DILATION}

Subglottic stenosis is treated with serial dilation by bougie (steel dilator).Its use is declining atpresent, and most surgeons prefer endoscopic balloon dilations. However, the results of bougie dilation are similar to the balloon dilation.

\section{TRACHEOSTOMY}

Tracheostomy (Fig. 3) is sometimesthesafest way to serve to airway in SGS. It may serve as a bridge before doing laryngotracheal surgery. Tracheostomy is important particularly when the larynx appears inflamed and active leading to failure of the surgery.

\section{OPEN SURGERY}

If the endoscopic approach fails or the appropriate success rate is not achieved, wide arrays of open airway reconstructive methods are performed to treat the laryngotracheal stenosis. Open airway laryngotracheal reconstruction can be done either as a single- or double-stage procedure. The single-stage procedure is not recommended in children with a history of difficult intubation, poor pulmonary function, reconstruction failure, or sedation problems [15]. In the double-stage procedure, first the patient requires tracheostomy, and the decannulation is done in a second stage.

\section{LARYNGOTRACHEAL RECONSTRUCTION}

Children with severe SGS benefit maximally from LTR by using anterior costal cartilage grafting, and it is also useful in failed cases of endoscopic management. Its success rate is more than $90 \%$ [16]. It can be a single- or double-stage procedure. The most common graft material used in LTR is costal cartilage, which gives excellent longterm outcomes [17]. The costal cartilage is readily available, easily craved, and easy to make into a flange so that it decreases the risk of graft prolapse into the laryngotracheal airway [17]. Sometimes the thyroid ala is harvested easily from same surgical field. It has the limitation of not being amenable to being craved with a flange. It is only harvested when a small graft is needed. Conchal cartilage of the pinna is also used for LTR, with successful outcome in one study [18].

\section{ANTERIOR CRICOID SPLIT}

It is an alternative procedure to tracheostomy in premature infants with prolonged intubation. It was initially started as an open surgical procedure to decompress the pressure of the endotracheal tube on the subglottic oedema. Presently, endoscopic anterior cricoid splits along with balloon dilations are reported with $83 \%$ success rate [19]. Larger studies are needed to establish this novel technique.

\section{CRICOTRACHEAL RESECTION}

The aim of this surgical procedure is to remove the stenotic segment of the airway, and the reunion of the healthy superior and inferior segments [20]. Cricotracheal resection is indicated in severe SGS or structurally inadequate subglottis and in patients who have undergone previous airway reconstructive surgery. The relative contraindications to CTR are low-grade SGS, stenosis within $3 \mathrm{~mm}$ of the vocal cords, or situations that impair mobilisation of the trachea, such as past history of distal tracheal surgery or injury to the tracheoesophageal septum [15]. Cricotracheal resection is a more technically challenging surgery in comparison to LTR, and there is disruption of the laryngeal framework and its supportive parts, no donor site morbidity, and decannulation may be successful. The disadvantages of CTR are the risk of catastrophic dehiscence of the repair and the chance of injury to the recurrent laryngeal nerve.

\section{SLIDE TRACHEOPLASTY}

Slide tracheoplasty done by extending through the anterior cricoid and effectively treating the patient with SGS. This approach may be cervical or transthoracic [21].

\section{STENTING}

Stenting with a Montgomery T-tube is relatively safe in children but is associated with aspiration in $11.5 \%$ and granulation in $16 \%$ of the cases [22]. The use of a silicone laryngotracheal mould reduces the formation of granulation, and it can stay for several months in difficult cases of SGS. The only prerequisite for this procedure is a tracheostoma. If excessive granulation tissue is seen on the cartilage graft after removal of the laryngotracheal mould (LT mould), mitomycin-C may be applied after removal of granulations and the mould can be reinserted for 1-2 months and an effective method for decannulation [23].

\section{POSTOPERATIVE COMPLICATIONS}

The complications after airway surgery may occur during surgery or after a procedure that includes desatu- 
ration, bleeding, wound infections, dehiscence, and graft dislodgment. The single-stage reconstruction of airways leads to additional complications like prolonged sedation/ paralysis, unplanned extubation, oedema in the airway, and severe narcotic withdrawal.

\section{CONCLUSIONS}

Subglottic stenosis is a challenging clinical entity. Inadequate airway due to SGS in children is a life-threatening situation. It is often good to avoid tracheostomy in children. After successful surgical treatment, most of the children are decannulated. An experienced team and advanced technology will be safest for obtaining optimum results.

\section{DISCLOSURE}

The authors declare no conflict of interest.

\section{REFERENCES}

1. Kim SM, Shin JH, Chang EY, et al. Slide thyrocricotracheoplasty for the treatment of high-grade subglottic stenosis in children. J Pediatric Surg 2010; 45: 2317-2321.

2. Choi SS, Zalzal GH. Changing trends in neonatal subglottic stenosis. Otolaryngol Head Neck Surg 2000; 122: 61-63.

3. Merostica PJ, Kuhl G, Carvalho PR. Association between length of the intubation and subglottic stenosis in children. Laryngoscope 2013; 123: 1049-1054.

4. Blanchard M, Leboulanger N, Thierry B, et al. Management specificities of congenital laryngeal stenosis: external and endoscopic approaches. Laryngoscope 2014; 124: 1013-1018.

5. Duynstee MLG, de Krijger RR, Monnier Ph, et al. Subglottic stenosis after endolaryngeal intubation in infants and children: result of wound healing processes. Int J Pediatr Otorhinolaryngol 2002; 62: 1-9.

6. Wei JL, Bond J. Management of endotracheal tube injury in neonate. Curr Opin Otolaryngol Head Neck Surg 2011; 19: 476-477.

7. Wasserzug O, De Rowe A. Subglottic stenosis: current concepts and recent advances. Int J Head Neck Surg 2016; 7: 97-103.

8. Meyer CM 3rd, O Connor DM, Cotton RT. Proposed grading system for subglottic stenosis based on endotracheal tube sizes. Ann Otol Rhinol Laryngol 1994; 103: 319-323.

9. Kelchner L, Baker Brehn S, de Alarcon A, et al. Update on pediatric voice and airway disorders: assessment and care. Curr Opin Otolaryngol Head Neck Surg 2012; 20: 160-164.

10. Swain SK, Sahu MC, Mohanty S, et al. Management of laryngotracheal stenosis - Still remains a challenge for successful outcome. Apollo Med 2016; 13: 102-107.

11. Cotton RT. Management of subglottic stenosis. Otolaryngol Clin North Am 2000; 33: 111-130.

12. Gharde P, Makhija N, Chauhan S. Post intubation tracheal stenosis in paediatric patients after cardiac surgery. Ann Cardiac Anaesthesia 2005; 8: 148-151.

13. Rutter MJ, Cohen AP, de Alarcon A. Endoscopic airway management in children. Curr Opin Otolaryngol Head Neck Surg 2008; 16: 525-529.

14. Lang M, Brietzke SE. A systemic meta-analysis of endoscopic balloon dilation of pediatric subglottic stenosis. Otolaryngol Head Neck Surg 2014; 150: 174-179.
15. Hart CK, Yang CJ, Rutter MJ. Reconstruction of the airway. In: Managing the difficult Airway: A Handbookfor Surgeons. Thompson JW, Vieira FO, Rutter MJ (eds.). JP Medical Publishers, London 2015; 115-124

16. Gustafson LM, Hartley BE, Liu JH, et al. Single stage laryngotracheal reconstruction in children: a review of 200 cases. Otolaryngol Head Neck Surg 2000; 123:430-434.

17. Jefferson ND, Cohen AP, Rutter MJ. Subglottic stenosis. Semin Pediatr Surg 2016; 25: 138-143.

18. Swain SK, Singh N, Samal R, et al. Use of conchal cartilages for laryngotracheal stenosis: experiences at a tertiary care hospital of eastern India. Indian J Otolaryngol Head Neck Surg 2016; 68: 445-450.

19. Mirabile L, Serio PP, Baggi RR, et al. Endoscopic anterior cricoid split and balloon dilation in pediatric subglottic stenosis. Int J Pediatr Otolaryngol 2010; 74: 1409-1414.

20. Jacquet Y, Lang F, Pilloud R, et al. Partial cricotracheal resection for pediatric subglottic stenosis: long-term outcome in 57 patients. J Thorac Cardiovasc Surg 2005; 130: 726-732.

21. de Alarcon A, Rutter MJ. Cervical slide tracheoplasty. Arch Otolaryngol Head Neck Surg. 2012; 138: 812-816.

22. Stern Y, Willging JP, Cotton RT. Use of Montgomery T-tube in laryngotracheal reconstruction in children: is it safe? Ann Otol Rhinol Laryngol 1998; 107: 1006-1009.

23. George M, Jaquet Y, Ikonomidis C, et al. Management of severe pediatric subglottic stenosis with glottic involvement. J Thoracic Cardiovasc Surg 2010; 139: 411-417. 